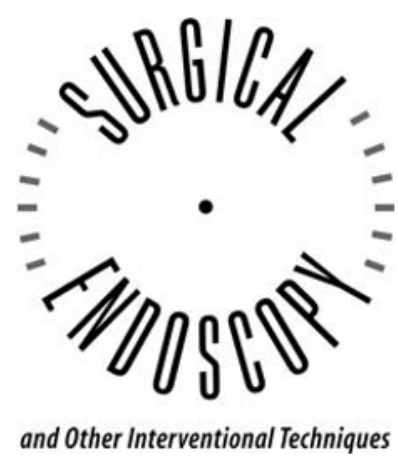

\title{
The author replies
}

We thank Dr.'s Lafullarde and Gys for their comments regarding "Risk factors and the prevalence of trocar site herniation after laparoscopic fundoplication." We completely concur with their comments and were unaware of their publication in 1999. We also encourage the widespread use of this technique and anecdotally can comment that since beginning the transrectus approach, we have markedly decreased if not eliminated the incidence of trocar herniation at the camera port site. We believe that the midline approach should be discouraged.

\section{J. H. Peters \\ D. J. Bowrey}

Departments of Surgery and Cardiothoracic Surgery University of Southern California University Hospital Los Angeles, CA 90033-4612, USA

Online publication: 26 March 2002 